hep-th/0409111, UTAP-495

\title{
Connecting black holes and black strings
}

\author{
Hideaki Kudoh ${ }^{1, *}$ and Toby Wiseman ${ }^{2}$, \\ 1 Department of Physics, The University of Tokyo, Bunkyo-ku, 113-0033, Japan \\ ${ }^{2}$ Jefferson Physical Laboratory, Harvard University, Cambridge MA 02138, USA
}

(Dated: September 2004)

\begin{abstract}
Static vacuum spacetimes with one compact dimension include black holes with localised horizons but also uniform and non-uniform black strings where the horizon wraps over the compact dimension. We present new numerical solutions for these localised black holes in 5 and 6-dimensions. Combined with previous 6 -d non-uniform string results, these provide evidence that the black hole and nonuniform string branches join at a topology changing solution.
\end{abstract}

PACS numbers: 04.50.+h, 04.25.Dm, 11.25.Mj

\section{INTRODUCTION AND SUMMARY}

The task of this letter is to resolve the structure of static vacuum solutions of Kaluza-Klein theory, namely pure gravity compactified on a circle. Let us firstly motivate our interests in this problem.

Many scenarios in string theory have extra dimensions large enough that they may be described geometrically 1, 2. In such models it is important to understand the behaviour and types of black hole solutions, and in particular whether there are potentially new signals from this physics [3].

The problem is also interesting as it is connected by holography to the phase structure of large $N_{c}$ super YangMills theory, compactified on a circle, at strong t'Hooft coupling [4, 5, 6, 7, 8, 9]. Since strong coupling results are scarce for this field theory, gravity provides the only window into this regime. Furthermore, the same phase structure predicted by gravity at strong coupling appears to persist to weak coupling [8].

Lastly, this letter provides evidence that for KaluzaKlein theory the 3 branches of solutions - the localised black holes, uniform and non-uniform strings - are connected in an elegant way, initially conjectured by Kol who postulated the problem is controlled by one relevant order parameter 10]. Learning more about this Morse-theory inspired approach may shed light on the new and exotic phenomena found in higher dimensions 11, 12, 13.

For small masses localised black holes (BH) should look like Schwarzschild solutions. Increasing their mass they deform as they feel the compact circle [14, 15]. The key question is then whether there is a maximum size for these solutions beyond which they no longer 'fit'.

In $d$ spacetime dimensions, with $d \geq 5$, uniform string (US) solutions exist. These are direct products of $(d-1)$ dimensional Schwarzschild with the circle, and are the only uncharged solution with horizon that is asymptotically $R^{1, d-2} \times S^{1}$ which has a simple analytic form (except for bubble spacetimes which we do not consider here [16]).

The non-uniform strings (NUS) were discovered when
Gregory and Laflamme showed that for a given circle size, uniform strings below a critical mass are linearly unstable [17, 18]. At the critical point there is a static mode breaking translation invariance on the circle. Motivated by dynamical considerations 19], Gubser showed this mode remains static to all orders in perturbation theory 20$]$.

Kol conjectured 10] that the 'waist' of the non-uniform string would shrink to nothing, locally forming a cone geometry, which connected to the black hole branch by resolving the cone to change the horizon topology (see also [21]). Elliptic numerical methods were used to construct the non-uniform strings in 6-d [22, 23] and the cone geometry was seen to emerge for the most non-uniform solutions 24]. These methods have recently been applied to construct the black hole solutions in 5-d and 6-d, and thus in principle we can test Kol's conjecture from the 'other side' 25, 26].

Here we present new numerical solutions for the 5-d and 6-d localised black holes which significantly improve on the previous works [25, 26] (see footnote 34]). In both dimensions they behave similarly, and we find a maximum size localised black hole that can 'fit' in the circle dimension. The 6 - $\mathrm{d}$ results provide evidence that the non-uniform and black hole branches do indeed merge at a topology changing solution.

\section{METHOD}

Both the non-uniform strings and black holes are static axisymmetric geometries that can be written in the form,

$$
d s^{2}=-e^{2 A} d t^{2}+e^{2 B}\left(d r^{2}+d z^{2}\right)+e^{2 C} r^{2} d \Omega_{d-3}^{2}
$$

where $A, B, C$ are functions of $r, z$. We take these to vanish at large radial coordinate $r$, and hence the geometry is asymptotically $R^{1, d-2} \times S^{1}$. The circle coordinate $z$ has length $L$ asymptotically. We then employ a numerical method developed in [22, 27, 28] which uses relaxation techniques to solve for $A, B, C$, whilst ensuring all the 


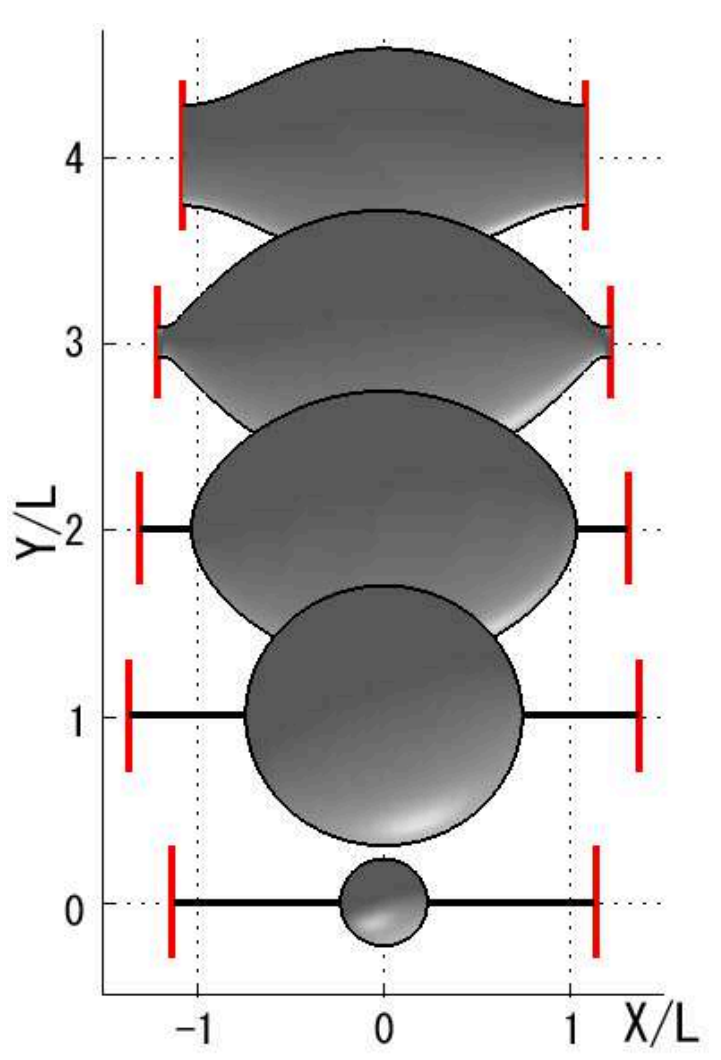

FIG. 1: Embeddings of the spatial horizon geometry of various 6-d BHs and NUSs in 5-d Euclidean space (suitably projected onto the page). For $\mathrm{BH}$ solutions we include the exposed symmetry axis in the embedding. The red vertical lines are to be periodically identified, generating the compact dimension.

Einstein equations are satisfied. The reader is referred to 22. for details of the procedure 35].

Following [29, 30, 31, 32], because the solutions are not asymptotically flat, they are characterised in terms of 2 asymptotic charges; the mass $M$ and a dimensionless tension $n$, which then give a first law, $d M=T d S+$ $n M d L / L$ where $T, S$ are the black hole temperature and entropy.

In the following plots, we will fix the asymptotic circle length $L=1$ for all solutions shown, and then use the quantity $n$ to characterise the solutions. For small black holes $n \simeq 0$, and for uniform strings $n=1 /(d-3)$. For convenience we normalise the thermodynamic quantities $T, S, M, n$ by their value for the critical uniform string, $T_{\text {crit }}, S_{\text {crit }}, M_{\text {crit }}, n_{\text {crit }}$.

The most error prone part of the numerical calculation is extracting the asymptotic form of the metric in order to compute $M, n$. Particularly difficult is $n$ as it is constructed from the difference of two relatively large quantities [25]. Here we compute both $n$ and $M$ using the first law and Smarr formula from $T$ and $S$, which are conveniently determined from the metric near the horizon.

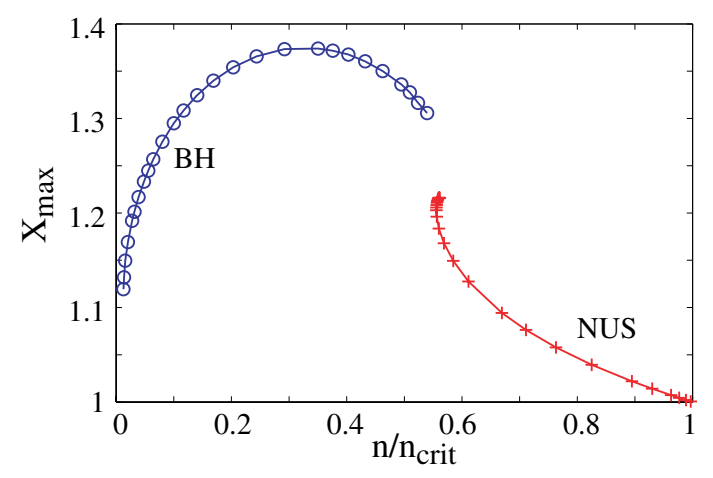

FIG. 2: Plot of $X_{\max }$ for 6-d NUSs and BHs, consistent with merger of the branches at $n / n_{\text {crit }} \simeq 0.55$.

\section{6-D RESULTS}

We now discuss the behaviour of 6 -d localised black holes, and the evidence that they join to the non-uniform branch. Firstly we consider the geometry of the horizon.

We may embed the spatial horizon geometry as a surface of revolution in 5-d Euclidean space. This is illustrated for several solutions in figure 1 The intrinsic geometry of the embedded surface is the same as that of the spatial sections of the 6 -d horizons. Note that for the black holes we also include the exposed rotational symmetry axis in the embedding plots. The Euclidean coordinate along the surface of revolution, $X$, should be thought of as periodic with $-X_{\max }<X<X_{\max }$ being the fundamental domain plotted. Then $X_{\max }$ gives a coordinate invariant measure of the 'size' of the geometry near the symmetry axis, and we plot this in figure 2

For non-uniform solutions we may compute the maximum and minimum radii of the horizon $R_{\max , \min }$. Analogously, for the black holes we have $R_{e q}$, the equatorial radius of the horizon, and $L_{a x i s}$, the proper length along the exposed symmetry axis. These are plotted in figure 3

From these graphs we see firstly that for increasing $n$ the equatorial radius of the black holes reaches a maximum and then starts to decrease, implying that there is indeed a maximum 'size' localised solution that can 'fit'. Secondly, from the horizon geometry it is plausible that the non-uniform and black hole branches merge around $n / n_{\text {crit }} \simeq 0.55$, where the value of $R_{e q}$ appears to tend to $R_{\max }$, and $X_{\max }$ is consistent with an extrapolation that agrees between the branches for this value of $n / n_{\text {crit }}$.

Now we consider thermodynamic quantities. In figures [4 and [5 we plot the entropy, temperature and mass of the solutions against $n$. Mirroring the behaviour of $R_{e q}$, we see the entropy and mass of the black holes reach a maximum and then decrease with increasing $n$. From this thermodynamic data we again clearly see evidence the branches merge.

Note that in our previous work 25] constructing the 6$\mathrm{d}$ black holes the solutions were only found in the regime 


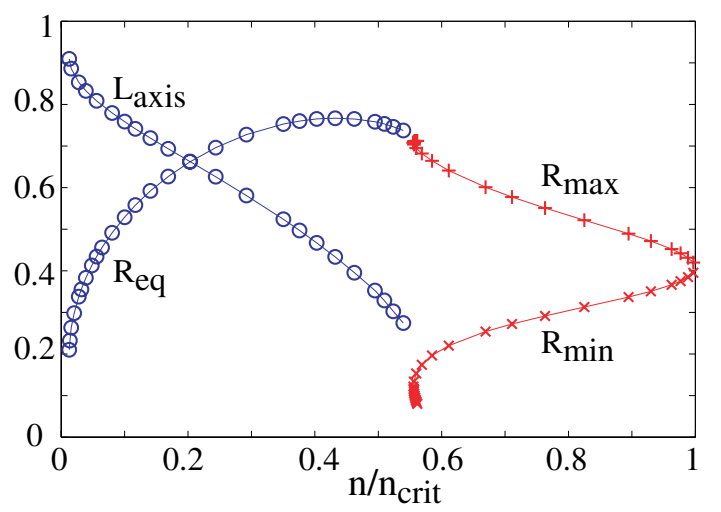

FIG. 3: Plot of horizon geometric quantities for 6-d solutions. Branches are consistent with a topology changing merger where both $L_{a x i s}$ and $R_{\min }$ go to zero, and $R_{e q}$ tends to $R_{\max }$. All solutions have $L=1$.
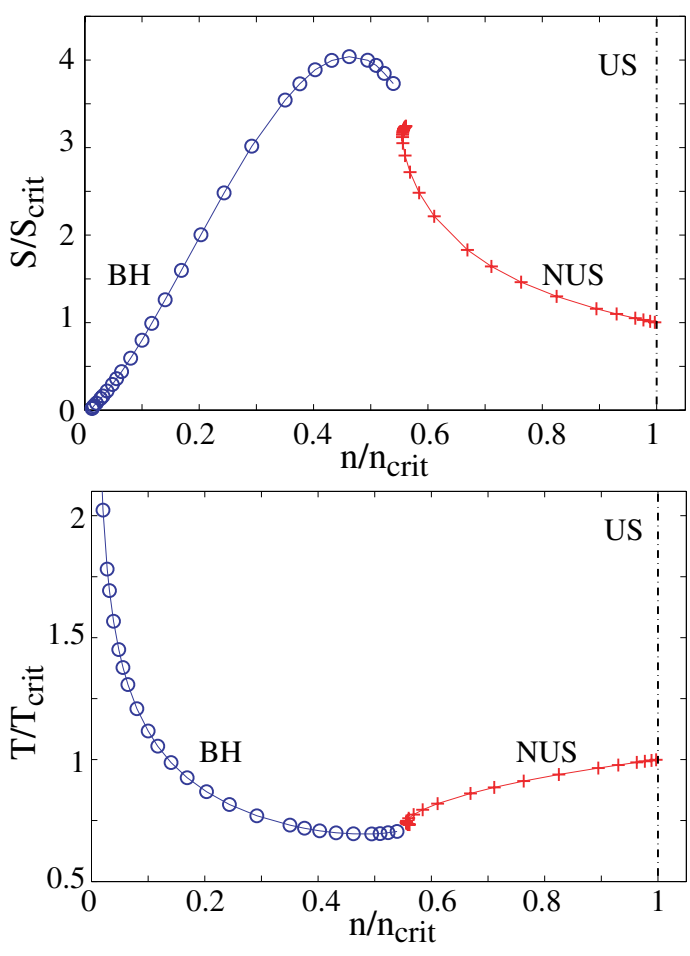

FIG. 4: Entropy and temperature for 6-d solutions.

where $R_{e q}$ and the mass were increasing with $n$, and hence it was not clear that the two branches could unify.

For very small masses the localised black holes are entropically favoured, and for very large masses only the uniform strings exist. In figure [6 we plot the entropy against mass for the 3 branches. We see uniform strings become entropically favoured, for a given mass, at masses above that of the critical uniform string, but below that of the maximum mass localised black hole.

Thus we see that the branches appear consistent with merger at $n / n_{\text {crit }} \simeq 0.55$. Let us now assume that this

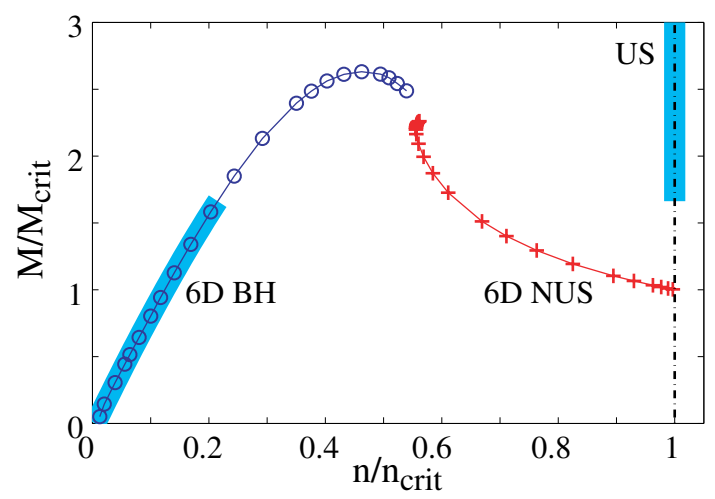

FIG. 5: Mass against $n$ for 6 -d solutions. The highlighting indicates which branch is entropically favoured for a given mass (see figure 6).

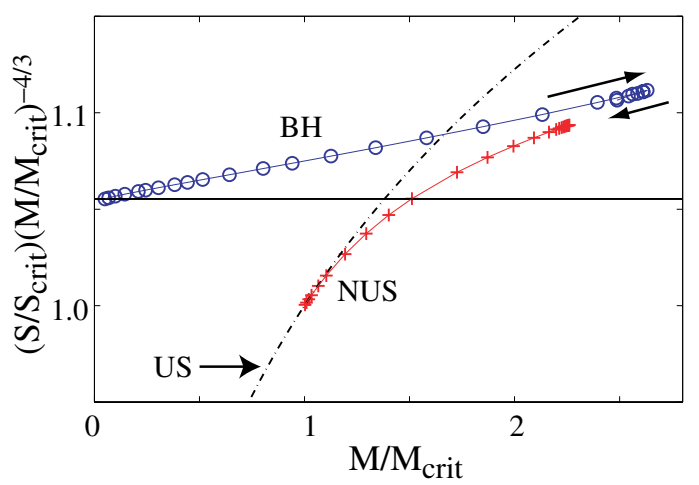

FIG. 6: Plot of $S M^{-4 / 3}$ against mass for the 6-d solutions. 6-d Schwarzschild behaviour is $S \propto M^{4 / 3}$ so represents a horizontal line. We see the localised BHs are entropically favoured for a given mass, for $M<1.7 M_{\text {crit }}$. Above this mass the uniform strings are favoured. The NUSs are never dominant.

occurs via a conical transition. We can then measure how far from the transition point the solutions are by estimating the geometric resolution of the cone. For the non-uniform strings this is given by the minimal radius of the horizon, $R_{\min }$, which for the most non-uniform string found in 22 was $R_{\min } \simeq 0.08$ in our units where $L=1$. The resolution for the black hole is given by the proper distance of exposed symmetry axis, $L_{\text {axis }}$. For these 6 -d solutions the smallest resolution found was $L_{\text {axis }} \simeq 0.27$. Therefore the most non-uniform strings found are still considerably closer to the assumed transition point than the most extreme localised black holes found here. For these non-uniform solutions the emergence of the cone geometry has been numerically demonstrated 24]. Repeating this for our new black hole solutions does indeed show an increase in curvature on the axis consistent with an emerging cone, but as the solutions are 'further' from the transition point this increase in curvature is still not large enough to be clearly distinguished from the background curvature of the black hole geometry. Thus whilst 


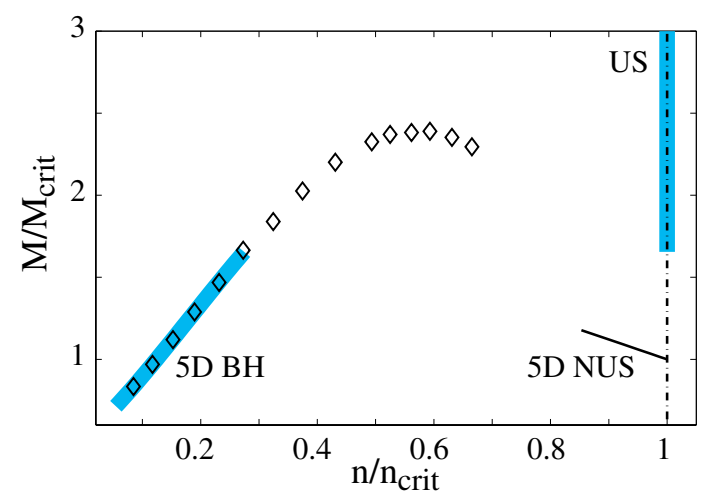

FIG. 7: Mass against $n$ for new 5-d localised BHs. Highlighting indicates entropically favoured solution at a given mass.

our new 6 -d black hole data is very suggestive the solution branches merge, it still cannot confirm that the detailed merger from the black hole side is via a conical transition. Indeed in Kol's original picture 10] we note that the cone may act only as an approximate local model for the merger, and the detailed behaviour very close to the point where the horizon pinches off may have a complicated behaviour that cannot necessarily be thought of as being smoothly resolvable.

\section{5-D RESULTS}

We now briefly discuss the 5 -d black hole solutions. All quantities behave in an analogous manner to their counterparts for the 6 -d solutions. Here we have simply plotted the mass against $n$ in figure 7 Note the mass increases past the critical uniform string mass with increasing $n$ (extending the previous numerical solutions in $5 \mathrm{~d}$ [26]), reaching a maximum and then decreases, again presumably to join the non-uniform branch. 36]

Acknowledgements We would like to thank Ofer Aharony, Gary Horowitz, Barak Kol, Jo Marsano and Shiraz Minwalla for enjoyable and enlightening discussions. TW was supported by the David and Lucille Packard Foundation grant number 2000-13869A. HK is supported by the JSPS. Numerical computations were carried out at the YITP and NAO.

* Electronic address: kudoh@utap.phys.s.u-tokyo.ac.jp

† Electronic address: twiseman@fas.harvard.edu

[1] N. Arkani-Hamed, S. Dimopoulos, and G. R. Dvali, Phys. Lett. B429, 263 (1998).

[2] I. Antoniadis, N. Arkani-Hamed, S. Dimopoulos, and G. R. Dvali, Phys. Lett. B436, 257 (1998).

[3] B. Kol (2002), hep-ph/0207037.
[4] N. Itzhaki, J. M. Maldacena, J. Sonnenschein, and S. Yankielowicz, Phys. Rev. D58, 046004 (1998).

[5] L. Susskind (1998), hep-th/9805115.

[6] E. J. Martinec and V. Sahakian, Phys. Rev. D59, 124005 (1999).

[7] M. Li, E. J. Martinec, and V. Sahakian, Phys. Rev. D59, 044035 (1999).

[8] O. Aharony, J. Marsano, S. Minwalla, and T. Wiseman (2004), hep-th/0406210.

[9] T. Harmark and N. A. Obers (2004), hep-th/0407094.

[10] B. Kol (2002), hep-th/0206220.

[11] R. Emparan and H. Reall, Phys. Rev. Lett. 88, 101101 (2002).

[12] E. Sorkin, Phys. Rev. Lett. 93, 031601 (2004).

[13] B. Kol and E. Sorkin (2004), gr-qc/0407058.

[14] T. Harmark, Phys. Rev. D69, 104015 (2004).

[15] D. Gorbonos and B. Kol, JHEP 06, 053 (2004).

[16] H. Elvang, T. Harmark, and N. A. Obers (2004), hepth/0407050.

[17] R. Gregory and R. Laflamme, Phys. Rev. Lett. 70, 2837 (1993).

[18] M. W. Choptuik et al., Phys. Rev. D68, 044001 (2003).

[19] G. Horowitz and K. Maeda, Phys. Rev. Lett. 87, 131301 (2001).

[20] S. Gubser, Class. Quant. Grav. 19, 4825 (2002).

[21] T. Harmark and N. Obers, JHEP 05, 032 (2002).

[22] T. Wiseman, Class. Quant. Grav. 20, 1137 (2003).

[23] T. Wiseman, Class. Quant. Grav. 20, 1177 (2003).

[24] B. Kol and T. Wiseman, Class. Quant. Grav. 20, 3493 (2003).

[25] H. Kudoh and T. Wiseman, Prog. Theor. Phys. 111, 475 (2004).

[26] E. Sorkin, B. Kol, and T. Piran, Phys. Rev. D69, 064032 (2004).

[27] T. Wiseman, Phys. Rev. D65, 124007 (2002).

[28] H. Kudoh, T. Tanaka, and T. Nakamura, Phys. Rev. D68, 024035 (2003).

[29] P. K. Townsend and M. Zamaklar, Class. Quant. Grav. 18, 5269 (2001).

[30] B. Kol, E. Sorkin, and T. Piran, Phys. Rev. D69, 064031 (2004).

[31] T. Harmark and N. A. Obers, Nucl. Phys. B684, 183 (2004).

[32] T. Harmark and N. A. Obers, Class. Quant. Grav. 21, 1709 (2004).

[33] B. Kleihaus and J. Kunz, Phys. Rev. Lett. 79, 1595 (1997).

[34] The black hole calculations were improved over our previous computation 25] largely by increasing the numerical resolution. In addition we discretise the lattice uniformly in $r$, rather than $r^{2}$, which increases the effective resolution near the symmetry axis. The new results reported used a maximum resolution of $256^{*} 1024$ points in the $r, z$ directions.

[35] See also 33] for work in 4-d utilising similar methods.

[36] We have been unable to extend the methods of 22] to construct this 5-d non-uniform branch. The method works with the asymptotic boundary at a relatively small radial coordinate location, but this boundary cannot be removed to large coordinate radius without an apparent numerical instability occurring - something that does not occur in 6 or more dimensions. 